El Computador en el Desarrollo de la Lectura en Inglés. San Juan de Pasto, Centro de Investigaciones Universidad Mariana: La Hormiga. ISBN: 978-958-96856-0-0. 1998.

APA citation style: Benavides B., Jorge. E. (1998). El Computador en el Desarrollo de la Lectura en Inglés. San Juan de Pasto: Centro de Investigaciones, Universidad Mariana. La Hormiga.

\title{
El Computador en el Desarrollo de la Lectura en Inglés
}

\author{
Jorge E. Benavides B. (joelbebu@gmail.com) \\ Departamento de Lingüística e Idiomas \\ Universidad de Nariño, San Juan de Pasto, Colombia
}

\section{Revisión de la literatura}

\section{Introducción}

La introducción de los computadores en educación ha sido en cierta medida impactante en muchas partes del mundo, en tal medida que ésta ha marcado un punto de partida para el replanteamiento de metodologías y especialmente de nuevas formas de pedagogía con bastantes y crecientes expectativas debido a algunos resultados iniciales halagadores. La preocupación de la gente en otros países en desarrollo ha tenido que ver con el hecho de que en su futura vida profesional el niño y el joven se van a encontrar ineludiblemente con la necesidad de tener que utilizar frecuentemente y en buena forma los equipos informáticos, sea cual sea su trabajo. El aprender a utilizarlos y explotarlos debidamente, justificaría con creces la inclusión de la asignatura de Informática para el aprendizaje de las ciencias básicas en cualquier sistema educativo. Esto se justificaría aún más si estas personas se van a preparar para ser docentes en cualquier nivel del sistema educativo.

En nuestro medio, la informática se encuentra en un proceso de introducción. Esta etapa es fundamental puesto que directamente va a determinar los estadios de familiarización, adaptación e integración de los computadores en el ámbito educacional, aunque se ve muy difícil acelerar el ritmo de cambio de nuestra educación para que deje de ser la cenicienta de nuestro sistema social. Sin embargo, y pese a todo lo que se ha pronosticado acerca de la aceptación de los computadores en el campo educativo y en el hogar como medios de instrucción, o mejor, como herramientas de aprendizaje, podemos atestiguar cómo cada día que pasa más instituciones educativas están adquiriendo, o en proceso de adquisición de sistemas de computación para el uso de estudiantes y profesores.

Ahora bien, la relación entre el bajo costo del computador comparado con las enormes servicios -cada día más crecientes- que puede llegar a prestar, hacen previsible que nuestro país se vaya poniendo a la altura de los países desarrollados como Gran Bretaña, Estados Unidos y Francia en este campo. Más aún, al contrario de lo que puede ocurrir con el usuario que compra un computador y luego lo olvida porque no sabe qué hacer con él (si no hay la debida 
El Computador en el Desarrollo de la Lectura en Inglés. San Juan de Pasto, Centro de Investigaciones Universidad Mariana: La Hormiga. ISBN: 978-958-96856-0-0. 1998.

capacitación), y por lo tanto ya no le interesaría oír más sobre informática, en educación, la implementación del uso de los computadores está haciéndose de manera lenta pero progresiva.

De todas maneras, lo que nos interesa a nosotros como educadores y formadores de educadores es darnos cuenta de las necesidades, intereses, problemas y aptitudes que nuestros hijos, estudiantes y futuros docentes tienen en este campo para entrar a tomar decisiones sobre la mejor forma de la introducción y uso de esta nueva tecnología. Cada día que pasa se hace más imperiosa la necesidad de conocer sobre la incidencia del computador en educación no solo por parte de profesores sino también por parte de la comunidad académica en conjunto. El futuro docente, por ejemplo, se encontrará con la ineludible necesidad de moverse en los campos de la tecnología de la información como un medio que le brinda la modernidad para el mejor desarrollo de su área y el de sus estudiantes; y a media que pasa el tiempo con mayor intensidad debido al carácter cambiante y vertiginoso de este campo.

Hoy en día, a pesar de que de la informática es bastante llamativa e interesante en nuestro medio, en realidad la introducción de este tipo de tecnología no ha tenido un camino aceptable a nivel de bachillerato y de universidad. En estos ámbitos, los computadores están siendo en gran medida subutilizados y empleados en tareas completamente diferentes para lo que fueron diseñados. Es pues importante manifestar la necesidad de realizar estudios de todo tipo, desde los de observación hasta los experimentales ya que los docentes tienen que concientizarse de que son los primeros y más llamados a mirar qué pasa en el contexto educativo con la utilización de los computadores que tantas expectativas está creando en la gente y que todavía cuenta con muchos escépticos, entre ellos, los mismos docentes (Benavides, 1989, 1993).

Los resultados de este estudio y sus implicaciones permitirán tener una idea bastante inicial acerca de las ventajas y/o desventajas del uso del computador como medio para suplementar el aprendizaje de un idioma extranjero como el inglés. Por otro lado podremos acercarnos al entendimiento de la interacción de los usuarios con la máquina: sus reacciones, interés, motivación, etc.

\subsection{Breve historia}

Siendo este aspecto bastante nuevo y cuya base teórica no ha sido apropiada completamente sino a nivel de experiencias iniciales, es necesario definir completamente nuestro campo de acción.

Este se origina en CALL (Computer-assisted Language Learning) Aprendizaje de Lenguas Asistido por Computador o redefinido como Informática Educativa para el Aprendizaje de los Idiomas, (INEDAl, Benavides 1993) o Aprendizaje de Lenguas Asistido por Computador (ALAC), que trata de la utilización de los computadores y de sus materiales informáticos para suplementar el aprendizaje de los idiomas. 
El Computador en el Desarrollo de la Lectura en Inglés. San Juan de Pasto, Centro de Investigaciones Universidad Mariana: La Hormiga. ISBN: 978-958-96856-0-0. 1998.

ALAC, en términos muy generales, puede entenderse como un concepto de utilizar el computador para propósitos de aprendizaje de lenguas. Podemos decir que ALAC comenzó como parte del proyecto educativo (PLATO) en los Estados Unidos, más específicamente en la Universidad de Illinois, uno de los sistemas más conocidos y más ambiciosos de los proyectos educativos que comenzaron en los 60's utilizando computadores centrales muy costosos. En Inglaterra los proyectos de tipo ALAC iniciaron durante la 70's, uno de los cuales comenzó en la Universidad de Hull con bastantes expectativas.

Durante la 70's con la llegada de microcomputadores económicamente más al alcance de la gente hubo muchos intentos, algunos exitosos, por introducir ALAC en instituciones educativas públicas y privadas, sin embargo el desarrollo del hardware (la máquina) estaba muchísimo más adelantado que el de la cantidad y calidad del software (programas) disponible. El auge de los microcomputadores en los 70's y 80's condujo a muchas instituciones a invertir en microcomputadores sin una planificación seria y sin base en necesidades concretas para lo que ellos querían hacer con estas máquinas. Este entusiasmo desbordado se convirtió en la regla en la mayoría de los países desarrollados con respecto al uso de este nuevo dispositivo en el campo educativo. Afortunadamente, la euforia descontrolada de la introducción de los computadores en educación parece haber disminuido a niveles más mesurados en estos días. A fin de cuentas, hay una lección para tenerse en cuenta y aprender de lo que sucedió en varios países desarrollados sobre la introducción de los computadores y principalmente en el aprendizaje de los idiomas, y es básicamente el hecho de que la introducción de la tecnología tiene que tomarse en forma más pragmática, pedagógicamente hablando, sobretodo en nuestro contexto.

Hoy ALAC se involucra con un tipo de actividades más comunicativas y la interacción usuario-computador, y usuario-usuario se dice es mucho más provechosa desde el punto de vista de la producción y más exigente a nivel cognitivo. Sin embargo, ALAC ha alcanzado una etapa en que la gente trata de encontrar un esquema pedagógicamente más adecuado para explicar los diferentes procesos que pueden establecerse o lograrse por medio del computador: materiales informáticos, y consecuentemente los diferentes escenarios y contextos de aprendizaje. En otras palabras, se necesita comenzar a establecer una pedagogía de base que no tenga que relacionarse necesariamente con las teorías metodológicas existentes. Frecuentemente las actividades en un contexto de ALAC, con el software o programas, la máquina misma, para no mencionar la interacción lograda dentro de un ambiente de ALAC, han sido cuestionadas desde el punto de vista de su valor pedagógico. Sin embargo, han existido muchos esfuerzos (aunque aislados) para introducir, filtrar y poner a disposición de una gran gama de usuarios la nueva tecnología en contextos educativos con resultados prometedores y esto es a veces ignorado por la mayoría de los críticos, o por parte de educacionalistas tecnófobos.

Según lo establecen Phillips (1985), y Weible (1987), (mencionados en Hubbard, 1992), ALAC y su tecnología han estado trabajando y buscando apropiarse afanosamente de una 
El Computador en el Desarrollo de la Lectura en Inglés. San Juan de Pasto, Centro de Investigaciones Universidad Mariana: La Hormiga. ISBN: 978-958-96856-0-0. 1998.

metodología adecuada y efectiva. A través de las diferentes etapas, los defensores de ALAC han propugnado por establecerlo como una disciplina por cuenta propia, y la abundante literatura apoya la necesidad de la apropiación de una estructura metodológica. Consecuentemente, se han realizado variados esfuerzos a fin de lograr esta meta. Higgins (1983, 1988), Underwood (1988), Phillips (1985), y Wyatt (1987), para mencionar algunos, y más recientemente Hubbard, (1992) han surgido con importantes ideas acerca de cómo puede establecerse una estructura metodológica para ALAC en el campo de programas para cursos y desarrollo de materiales.

Como sucede en el campo de la instrucción en el aula de clase, no son los materiales, los elementos más importantes sino lo que el profesor y los estudiantes hacen con ellos, es decir el uso que se les da. En el campo del Aprendizaje de Lenguas Asistido por Computador podríamos aplicar el mismo principio; como fue el caso de los principios de ALAC el computador solo fue utilizado como parte de los materiales audiovisuales que ayudaban al profesor principalmente en tareas magistrales de instrucción: una tarea considerada hoy en día, realmente limitante.

\subsection{Materiales utilizados en ALAC}

Estos los conforman la gran cantidad de software diseñado comercialmente por firmas comerciales, o por programadores, instituciones educativas, profesores, y una mínima parte por pedagogos, para suplementar la instrucción del idioma Inglés.

Existe una gran cantidad de tipos de programas, muchos de los cuales se diferencian entre si por la clase de actividad que originan. De esta manera y partiendo de la base de que el computador puede ser una herramienta educativa bastante versátil, tiene posibilidades de generar una amplia variedad de actividades, entre las cuales se encuentran las de los programas de construcción de texto, juegos educativos, ejercicios o pruebas de contenidos específicos, simulación, solución de problemas, exploración, diálogos interactivos, etc.

La simulación se entiende aquí como cualquier actividad de aprendizaje que busca recrear aspectos y contextos de la vida real. En este caso lo que el computador aporta es la habilidad de modelar sistemas complejos aplicados a diferentes situaciones de aprendizaje. El usuario, no tiene que preocuparse por realizar cálculos de los resultados de una acción, el computador lo hace por él, en un tiempo mínimo, con gran precisión, y proporcionando corrección y retroalimentación instantáneas. La actividad del usuario es la toma de decisiones sobre el curso de una acción, en una actividad de simulación, por ejemplo.

\subsection{Programas de reconstrucción de texto}

QUARTEXT, de Longman Group, 1988 es un programa que ofrece varias posibilidades de ejercicios, uno de los cuales, (CHEAT), involucra al usuario en una competencia con la máquina para ver quien reconstruye más texto, recibiendo puntos por cada palabra y puntos extras cuando el usuario decide que el computador ha sido cogido infraganti colocando una 
El Computador en el Desarrollo de la Lectura en Inglés. San Juan de Pasto, Centro de Investigaciones Universidad Mariana: La Hormiga. ISBN: 978-958-96856-0-0. 1998.

palabra que a pesar de que encaja con los espacios de la palabra, no concuerda con el contexto. Como QUARTEXT, hay una gran cantidad de programas utilizados con éxito en la instrucción suplementaria del idioma Inglés, ya sea en el campo del vocabulario o la comprensión de lectura.

Para dar una idea de la disponibilidad de software se proporcionará a continuación una lista de tipos de programas desarrollados para la lectura y el vocabulario. La siguiente es una muestra, muy pequeña por cierto, de programas de ALAC según la habilidad y la actividad que ellos promueven.

\subsection{Vocabulario}

Jones (1987) ofrece algunas sugerencias sobre disponibilidad y de cómo utilizar programas para el aprendizaje de vocabulario. Este tipo de programas frecuentemente se considera altamente atractivo (validez física) y motivante para los aprendices ya que la mayoría de ellos introducen algún tipo de formato de juego. Los siguientes son simplemente algunos de los programas de vocabulario más ampliamente difundidos.

\section{- CRUCIGRAMA}

Es uno de los programas más ampliamente conocidos y funciona en forma similar a los crucigramas de texto impresos. Dependen básicamente de la capacidad del profesor en crear estas actividades con el vocabulario foco y para que el estudiante los reconstruya.

\section{- HANGMAN (El ahorcado)}

En este programa el aprendiz trata de descubrir la palabra oculta introduciendo diferentes letras. Uno de los juegos de vocabulario más populares del cual hay tantas versiones como escritores, desarrolladores de juegos y profesores que trabajan con paquetes de autoría (para adecuar los contenidos de los programas).

\section{- ALPHAGAME}

Un juego de ordenamiento alfabético en el que el aprendiz tiene que 'adivinar' la palabra que el computador 'piensa' escribiendo palabras y averiguando si se encuentra ya sea antes o después en el diccionario con base en la palabra foco que provee el computador.

\section{- WORDSQUARES}

El aprendiz tiene que extraer las palabras (de arriba abajo, o diagonalmente) a partir de un cuadro con palabras. Algunas letras o palabras pueden también estar ocultas en el cuadro. Algo similar al conocido juego de sopa de letras.

\section{- MINDWORD}

Este es un tipo de programa más sofisticado en que el aprendiz tiene que adivinar la palabra que el computador 'piensa' suministrándole indicios o pistas semánticamente contextualizadas sobre la palabra. 
El Computador en el Desarrollo de la Lectura en Inglés. San Juan de Pasto, Centro de Investigaciones Universidad Mariana: La Hormiga. ISBN: 978-958-96856-0-0. 1998.

\section{- SCRABBLE}

Es un juego de computador muy efectivo que comúnmente incluye alrededor 20.000 palabras incorporadas en su diccionario para la construcción del juego con la posibilidad de introducir muchas más y por ende con posibilidades de utilizarse como una herramienta de autoría.

\subsection{Los multimedios}

Los multimedios o MULTIMEDIA es uno de los últimos desarrollos en la tecnología del computador y se basa en el concepto de la integración de sonido (voz humana incluida, hasta sonido estéreo de alta calidad), video, gráficos y texto. Este tipo de medios diferentes y sin embargo complementarios se obtienen a partir de discos compactos con tecnología láser cuya base es un computador de tipo medio equipado con una unidad de disco compacto (CD-ROM).

Este nuevo desarrollo en la tecnología del computador presenta tanto a profesores como a estudiantes un amplio número de oportunidades tanto desde el punto de vista de la enseñanza magistral como el del aprendizaje implícito o indirecto. Sin embargo, este tipo de recursos no está aún al alcance de todas las instituciones educativas de nivel básico primario o secundario. Por otro lado, todavía existe incertidumbre sobre cuán efectivo sea este tipo de tecnología para que pueda contribuir realmente en el mejoramiento del aprendizaje de una lengua.

Hay por supuesto muchas otras posibilidades para el aprendizaje de la gramática, del vocabulario e inclusive de patrones discursivos a partir de tipos diferentes de software especialmente con programas que utilizan la Multimedia donde la integración de sonido, video, gráficas, y texto parece proveer oportunidades más interactivas para aprender. Consecuentemente, se piensa que este tipo de actividades recreadas por medio del computador podría ayudar a desarrollar diferentes estilos y estrategias de aprendizaje.

\subsection{Características de los materiales en ALAC}

Las siguientes son algunas de las características más comunes de los programas de computador utilizados en ALAC, que se requieren para el uso y manejo consistentes de los mismos y para que la evaluación de resultados se haga en forma más confiable.

1. Producción automática de ejercicios.

2. Suministro de corrección y retroalimentación.

3. Desarrollo interactivo de las sesiones entre el usuario y el computador.

4. Suministro de resultados y análisis de los mismos después de determinada sesión.

5. Utilización amistosa (user-friendly) del programa (eliminando las ambigüedades en lo posible)

6. Suministro de opciones diversas dentro del programa (selección de ejercicios varios, acceso a las respuestas, acceso a pistas, opción para detenerse, continuar, o terminar)

7. Presentación visual atractiva. 
El Computador en el Desarrollo de la Lectura en Inglés. San Juan de Pasto, Centro de Investigaciones Universidad Mariana: La Hormiga. ISBN: 978-958-96856-0-0. 1998.

8. Disponibilidad de niveles de dificultad.

9. Dirección personalizada.

10. Presentación de una interface intuitiva (que anime al usuario a intentarlo aún sin conocer a fondo los comandos)

Programas con inclusión de aspectos tecnológicos más avanzados y que se requieren en determinadas instituciones, hacen uso de voz sintética y/o digitalizada para proveer corrección o input auditivo al usuario en un contexto de aprendizaje.

\subsection{Aspectos pedagógicos e investigativos de ALAC}

Underwood (1986) pone de manifiesto que a diferencia de actividades de tipo conductista como los de práctica extensiva existen innovaciones en el campo de la metodología comunicativa de la lengua en ALAC. Así, propone que las características de estos programas comparadas con las de las no comunicativas son:

\section{Aspectos comunicativos de programas en ALAC}

-No impone secuencia gramatical determinada.

-Proporciona pistas útiles.

-El estudiante se encuentra en control de las actividades.

-El estudiante se relaciona con los contenidos de la materia de manera personal.

-El estudiante crea su propia experiencia de aprendizaje.

-El estudiante percibe la tarea como un suplemento motivante de la clase.

-El estudiante percibe la tarea como una actividad novedosa

\section{Aspectos No-comunicativos de programas en ALAC}

-Incorpora secuencia gramatical.

-Informar al estudiante de sus errores.

-El programa está en control de las actividades.

- Los contenidos de la materia son irrelevantes para los estudiantes.

-Las lecciones están predeterminadas.

-El estudiante percibe la tarea como una lección obligatoria.

-El estudiante percibe la tarea como idéntica a las del aula de clase.

El aprendizaje de la lengua asistido por computador busca formas de facultar a los individuos para dirigir su propio ritmo de aprendizaje, en vez de ceder control sobre éste a una entidad autoritaria, como sucede en los modelos conductistas o magistrales. A continuación podemos encontrar las características que expone Higgins (1988) sobre la diferencia entre Maestro y Pedagogo relacionada con el papel de computador.

\section{El Maestro:}

-Dirige el aprendizaje de los estudiantes.

-Tiene autoridad para evaluar, elogiar, censurar. 
El Computador en el Desarrollo de la Lectura en Inglés. San Juan de Pasto, Centro de Investigaciones Universidad Mariana: La Hormiga. ISBN: 978-958-96856-0-0. 1998.

-Estructura el orden de los eventos.

-Explica reglas, da ejemplos.

-Repite interminablemente.

El pedagogo:

-Ayuda en el aprendizaje de los estudiantes.

-No ejerce autoridad para censurar.

-No provee ningún tipo de estructura.

-Contesta las preguntas de los estudiantes.

-Sigue las sugerencias de los estudiantes.

\subsection{ALAC y el aprendizaje humanístico}

Papert (1980) toma el enfoque humanístico del aprendizaje, como el postulado por Rogers, (1961) y lo adapta al concepto del aprendizaje basado en el apoyo que presta el computador. Este último considera que en un ambiente libre de intimidaciones el individuo aprende a ser libre y se sumerge en un proceso educativo ideal en el que el aula llega a ser tan efectiva como una clínica (Rogers, 1969). En otras palabras, este tipo de ambiente depende de la relación entre el facilitador y el cliente, con tres cualidades importantes. La primera es la que Rogers (1961) llama 'la transparencia de mi parte' la cual se interpreta en el área del aprendizaje de la lengua, como un foco:

...la meta de la educación es la facilitación del cambio y del aprendizaje... aprender a aprender es más importante que el hecho de que a alguien se le 'enseñe' algo, lo cual generalmente proviene de la posición del profesor quien decide unilateralmente que debe enseñarse. (Brown, 1980) (citado en Pennington, (1992:13).

La segunda cualidad es la del terapista, que se formula como la 'preocupación positiva e incondicional para con el cliente' y la tercera es la del 'entendimiento condescendiente: el deseo continuo de comprender los sentimientos, experiencias y manifestaciones significativas y personales del cliente'. Estas son las características del educador humanista cuyo interés es el de propiciar ambientes que conduzcan al aprendizaje y el cual también puede eventualmente llevarse a efecto mediante el sistema de aprendizaje asistido por computador.

El desarrollo reciente de ALAC ejemplifica los paradigmas humanistas, por ejemplo, cuando la gente dice que ALAC puede llegar a ser más 'amistoso' significa que los programas para cursos tienden estos días a ser más lo que los estudiantes quieren que sean, que para lo que un diseñador o programador en particular pudiera haber destinado originalmente su producto. Esto ha conducido a reconocer en el computador y los programas (hardware y software conjuntamente) una ayuda para aprender, o como una herramienta de aprendizaje, o mucho mejor como un informante de la lengua más que como un instructor programado. 
El Computador en el Desarrollo de la Lectura en Inglés. San Juan de Pasto, Centro de Investigaciones Universidad Mariana: La Hormiga. ISBN: 978-958-96856-0-0. 1998.

La consideración anterior podría tomarse en cuenta cuando se discute sobre la conveniencia o no de la introducción e integración de ALAC en el currículo educativo como un medio que permita a los estudiantes experimentar con conceptos enseñados en otra circunstancia o en otro lugar (tiempo y espacio). Por otro lado, siendo parte de cualquier curso de lengua, ALAC puede también considerarse como algo que el estudiante puede emplear sobre una base adhoc o de 'libre-acceso' sin que necesariamente sea parte de un curso de estudio establecido (laboratorio de computadores o centro de recursos)

Es especialmente importante mencionar que a pesar de ser un campo nuevo y que usualmente se empieza a aplicar antes de investigar -lo cual parece ser una constante en todas las ciencias y artes- se han propiciado algunos intentos por desarrollar estudios de carácter experimental en nuestro país. Henao y Giraldo (1991) en la Universidad de Antioquía realizaron un primer intento en esta área tratando de evidenciar la cantidad y calidad de las composiciones escritas en Español realizadas por niños entre 11 y 13 años utilizando los computadores como una herramienta de aprendizaje y un procesador de texto que les permitía desarrollar la habilidad de la escritura. Los sujetos tuvieron la oportunidad de mostrar diferencias significativas al producir sus textos en el computador a diferencia de lo que hicieron en forma manuscrita. Estos resultados son particularmente importantes puesto que proporcionan una evidencia más de la forma cómo en realidad el computador puede ser útil, cómo puede ayudar en el campo pedagógico convirtiéndose en una herramienta versátil, novedosa, interesante y productiva en tanto el participante pueda manipularlo y el primero le brinde las diferentes posibilidades de aprendizaje a su alcance.

Ha existido mucha especulación, sobre todo a nivel internacional, sobre el uso de los computadores en educación desde hace ya dos décadas. Muchos cuestionamientos se han hecho acerca de las ventajas del uso de los computadores en el aula de clase (Kinzer, 1986). Sin embargo, hay muchas expectativas acerca del efecto que estos puedan causar en cuanto a la atención y la motivación en los estudiantes jóvenes (White, 1983).

A nivel internacional, y a pesar de que se ha realizado alguna clase de investigación incipiente, aclarando que aún se necesita una estructuración de la misma en este campo, la mayor parte de la evidencia acerca de la ventaja de los computadores como una forma alterna de enseñanza en educación es de tipo anecdótico y personal. Y a pesar de que en la opinión de los expertos y según resultados de algunas investigaciones pioneras que se orientan a promover el uso de los computadores en la enseñanza de la lectura, muy pocos estudios se han realizado acerca de la diferencia entre la instrucción y el aprendizaje (Blair y Rupley 1986).

En algunos estudios recientes las investigaciones se han concentrado en algunas de las implicaciones del uso de los computadores en el proceso de lectura (atención, culminación de tareas, y comprensión). Belmore, $(1983,1985)$ encontró que estudiantes de nivel universitario se demoraron más y comprendieron menos utilizando la presentación en el computador, en actividades de lectura, a diferencia de lo que lograron sobre el papel. Zuk, (1986) al trabajar con 
El Computador en el Desarrollo de la Lectura en Inglés. San Juan de Pasto, Centro de Investigaciones Universidad Mariana: La Hormiga. ISBN: 978-958-96856-0-0. 1998.

niños también encontró que el mayor efecto en cuanto a la presentación del texto para la lectura (texto en papel y texto en la pantalla del computador) se encontraba en el aspecto de la terminación de la actividad de la lectura. Los niños se demoraron más leyendo desde el computador que desde el papel.

En cuanto al aspecto de la correlación entre la velocidad y la comprensión, (Benavides, 1997) encontró una positiva correlación entre la velocidad y la comprensión en Inglés técnico e inclusive algo mayor entre la velocidad y el aprendizaje de vocabulario (reconocimiento), utilizando estudiantes de nivel universitario. Eso, ocurrió de manera interesante, sobretodo en el post-test, donde se pudo apreciar que el tratamiento sobre lectura rápida representaba niveles de correlación positiva entre las variables mencionadas.

\section{Bibliografía}

Belmore, S. (1985). Reading Computer-presented Text. Bulletin of the Psychonomic Society. 23(1), 12-14..

Benavides B., Jorge E. (1989). Iniciación a la Instrucción Complementaria del Inglés mediante Computadores. Revista de Investigaciones. Universidad de Nariño. No. 5, vol. III. 179-193.

Benavides B., Jorge E. (1993). Informática Educativa para la Enseñanza de los Idiomas. Revista de Investigaciones. Universidad de Nariño. Año 7, No. 1, vol. Vl. 196-206..

Benavides B., Jorge E. (1997). La lectura rápida en el desarrollo de la velocidad y la comprensión en Inglés. Universidad de Nariño, Pasto: Editorial Universitaria. UNED.

Blair, F. L., Rupley, W. \& Jones M. (1986). Microcomputers: Another False Prophet?. Reading Research and Instruction. 26(1), 58-61..

Brown, H. D. (1980). Principles of language learning and teaching. Englewood Cliffs, HJ: Prentice-Hall.

Henao. O, \& L. Giraldo (1991). El Computador en la Enseñanza de la Escritura. Medellín (Universidad de Antioquia): Textos y Diseños.

Higgins, J. (1983). Can computers teach?, CALICO Journal, 1: 2, 4-6

Higgins, J. (1988). Language, Learners and Computers. London: Longman.

Hubbard, P. (1992). A Methodological Framework for CALL: Courseware Development. In M. Pennington, \& V. Stevens. (eds.). Computers in Applied Linguistics. Clevedon: Multilingual Matters Ltd.

Jones, C. \& Sue Foretscue. (1987). Using Computers in the Language Classroom. London: Longman.

Kinzer, Ch. (1986). A 5 Part Categorization for Use of Microcomputers in Reading Classrooms. Journal of Reading. 30(3), 56-76..

Papert, S. (1980). Mindstorms: Children, Computers, and Powerful Ideas. New York: Basic Books.

Philips, M. (1985). Logical possibilities and classroom scenarios for the development of CALL. In C. Brumfit, M. Philips \& P. Skehan (eds.) Computers in English Language Teaching. New York: Pergamon. 
El Computador en el Desarrollo de la Lectura en Inglés. San Juan de Pasto, Centro de Investigaciones Universidad Mariana: La Hormiga. ISBN: 978-958-96856-0-0. 1998.

Rogers, C. (1961). On Becoming a Person: A therapist's View of Psychotherapy. London: Constable.

Rogers, C. (1969). Freedom to learn. Colombus, OH: Chas. E. Merrill Publishing Co.

Underwood, J. (1984). Linguistics, Computers and the Language Teacher: A Communicative Approach. Rowley, MA: Newbury House.

Weible, D. (1987). Towards a Media-specific methodology for CALL. In W. Flint Smith (ed.), Modern Media in Foreign Language Education: Theory and Implementation. Lincolnwood, IL: National Textbook Company.

White, M. (1983). Synthesis of Research on Electronic Learning. Educational Leadership. 40(8), 13-15.

Windeatt (1986). Observing CALL in action, in G. Leech \& C. Candlin (eds), Computers in English Language Teaching and Research. London and New York: Longman.

Wyatt, D. (1983). Three major approaches to developing computer-assisted language learning materials for microcomputers. CALICO Journal, 1: 2, 34-38.

Zuk. D. (1986). The Effects of Microcomputers on Children's Attention to Reading. Computers in the Schools. (3), 39-51. 